\title{
Epidermal restriction confers robustness to organ shapes
}

\author{
Lüwen $\mathrm{Zhou}^{1 \dagger} *$, Fei $\mathrm{Du}^{2 \dagger}$, Shiliang Feng', Jinrong $\mathrm{Hu}^{3,4}$, Shouqin $\mathrm{Lü}^{3,4}$, Mian Long ${ }^{3,4}$ and Yuling Jiao ${ }^{2,4}$ \\ 1. Smart Materials and Advanced Structure Laboratory, School of Mechanical Engineering and Mechanics, Ningbo University, Ningbo 315211, \\ China \\ 2. State Key Laboratory of Plant Genomics, Institute of Genetics and Developmental Biology, Chinese Academy of Sciences, Beijing 100101, \\ China \\ 3. Key Laboratory of Microgravity (National Microgravity Laboratory), Center of Biomechanics and Bioengineering, and Beijing Key Laboratory \\ of Engineered Construction and Mechanobiology, Institute of Mechanics, Chinese Academy of Sciences, Beijing 100190, China \\ 4. University of Chinese Academy of Sciences, Beijing 100049, China \\ ${ }^{\dagger}$ These authors contributed equally to this work. \\ *Correspondence: Lüwen Zhou (zhoulvwen@nbu.edu.cn)
}

doi: 10.1111/jipb.12998

\begin{abstract}
The shape of comparable tissues and organs is consistent among individuals of a given species, but how this consistency or robustness is achieved remains an open question. The interaction between morphogenetic factors determines organ formation and subsequent shaping, which is ultimately a mechanical process. Using a computational approach, we show that the epidermal layer is essential for the robustness of organ geometry control. Specifically, proper epidermal restriction allows organ asymmetry maintenance, and the tensile epidermal layer is sufficient to suppress local variability in growth, leading to shape robustness. The model explains
\end{abstract}

the enhanced organ shape variations in epidermal mutant plants. In addition, differences in the patterns of epidermal restriction may underlie the initial establishment of organ asymmetry. Our results show that epidermal restriction can answer the longstanding question of how cellular growth noise is averaged to produce precise organ shapes, and the findings also shed light on organ asymmetry establishment.

Edited by: Juan Dong, Rutgers the State University of New Jersey, USA

Received Jun. 17, 2020; Accepted Jul. 28, 2020; Online on Jul. 29, 2020

\section{INTRODUCTION}

The three-dimensional (3D) forms of organs are highly reproducible insofar as the same shape is achieved regardless of intrinsic and extrinsic perturbations. The remarkable robustness of organ shaping is evident in both plants and animals and represents a central question in biology (Lander 2011). However, the accumulated evidence indicates that cells within organs are often variable. For example, stochastic gene expression occurs in various plant organs (Araújo et al. 2017; Han et al. 2017), and neighboring cells of similar identity may grow at different rates (Hong et al. 2018).

Gene expression randomness and subsequent cell fate divergence can be a driving force for pattern formation. For example, gene expression level stochasticity in sepal and leaf epidermis can induce giant cells, which derive from endoreduplication events (Meyer et al. 2017). In terms of organ formation, it has been proposed that the initial patterning may result from fluctuations in auxin levels. For example, a region with randomly higher auxin levels would induce further auxin accumulation, which would in turn lead to the formation of the first lateral organ from the shoot apical meristem (de Reuille et al. 2006; Jönsson et al. 2006; Smith et al. 2006). The initial lateral organ determines the pattern of subsequent lateral organ formation. Similar auxin level fluctuations at the leaf margin may also explain leaf margin serrations in 
simple leaves and leaflet patterning in compound leaves (Bilsborough et al. 2011). Thus, stochasticity is a driving force for a wide range of patterning processes. At the same time, organ shape robustness is inherent to most organs. Therefore, how is organ shape robustness achieved while virtually all genes have stochastic expression across cells? Recent works have started to shed light on this fundamental question. For example, it was recently shown that reproducible sepal shape requires a balanced amount of reactive oxygen species (Hong et al. 2016). In addition, mechanical signals may also contribute to organ shape reproducibility by isolated rapidly growing cells and limit their imapct on organ shape (Hervieux et al. 2017). However, it is presently unknown if there is a conserved mechanism that ensures organ shape reproducibility across a wide range of organs with distinct shapes.

The importance of the epidermal cell layer for growth has long been recognized, with renewed focus on its role in recent years (Kutschera and Niklas 2007). This idea, iconized as the "tensile skin" theory or "epidermal-growth-control" theory, proposes that the peripheral thickened outer cell walls, together with the cuticle of some aerial organs, confer a higher bending stiffness of the epidermis layer than inner tissue layers and determine the rate of organ growth (Onoda et al. 2015; Galletti et al. 2016). Recent theoretical works that considered the balance between epidermal and inner cells showed that the final organ shape and size rely on the presence of a stiff epidermis that is limiting for growth (Boudon et al. 2015). Meanwhile, experimental evidence suggests that the levels of brassinosteroid phytohormones in the epidermis determine wall stiffness (Wolf et al. 2012) and organ size (Savaldi-Goldstein et al. 2007).

In the present study, we investigated how restriction by the epidermal layer affects the robustness of organ shape. Using mechanical modeling, we showed that a proper mechanical restriction by the tensile epidermal layer is necessary for consistent organ shaping. Epidermal restriction averages variations in cellular growth, which may otherwise self-amplify. The role of epidermal restriction in shape robustness is applicable to a wide range of plant organogenesis processes.

\section{RESULTS}

\section{Computational framework for modeling organ shapes}

We developed a two-dimensional (2D) computational model. As illustrated in Figure $1 \mathrm{~A}$, a typical vascular plant organ includes outer cell walls, epidermal cells, and inner cells from the outside to the inside. In our modeling system, we used a vertex model to simulate the growth and division of epidermal cells and inner cells (Figure 1B). Since it has been well known that the outer wall of an epidermal cell is much thicker than its inner wall and the wall of inner cells in plant organs like shoot apical meristem (Kierzkowski et al. 2012) and leaf (Onoda et al. 2015; Zhao et al. 2020), the outer cell walls of epidermal cells were assigned springs three times stiffer than those representing inner walls. The shape of a cell is described by a set of vertices that represent the common point of two or more neighboring cells. Cellular interfaces and cell volumes (cell volume is simplified as area in 2D model) can be defined from the positions of the vertices.

Cell expansion is driven by turgor pressure, and the balance between turgor pressure and wall resistance determines the cell size. Growth of a cell is reinforced along the minimum stress direction, while the cell division plane is oriented along the maximum stress direction (Uyttewaal et al. 2012; Louveaux et al. 2016) (Figure 1C). In our model, the cell growth is achieved by increasing the natural lengths of the wall springs associated with the growing cell, and the cell division is orientated along the direction of maximal tensile stress according to experimental results (Louveaux et al. 2016). Our modeling framework is solved numerically with the energy minimization method which allows convenient simulations of different cell division modes (Figure 1D).

\section{Proper epidermal constraints ensure organ shape anisotropy}

To analyze the relationship between organ shape development and epidermal restriction, we investigated a series of conditions with the restrictive effect of the epidermal layer applied at different levels. We first analyzed the effect of epidermal restriction on anisotropic organ shape maintenance, which is commonly seen in nature. We simulated organs with different levels of shape anisotropy, which were represented by different initial aspect ratios for the long axis over the short axis 

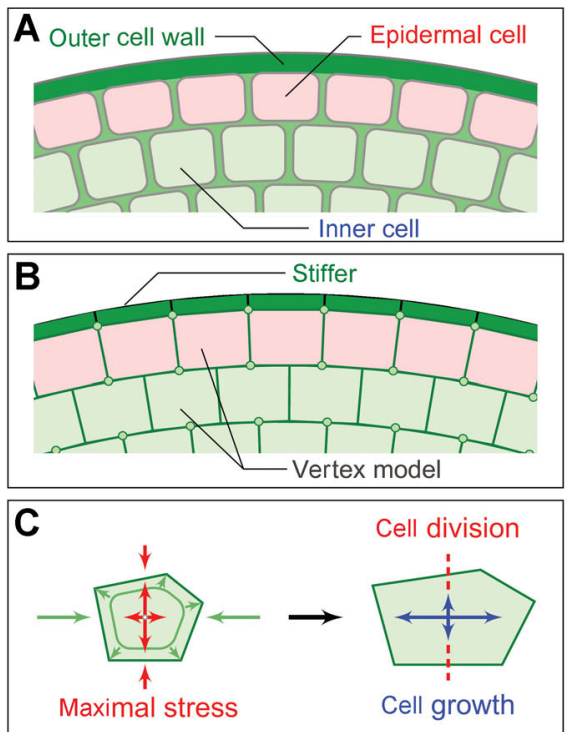

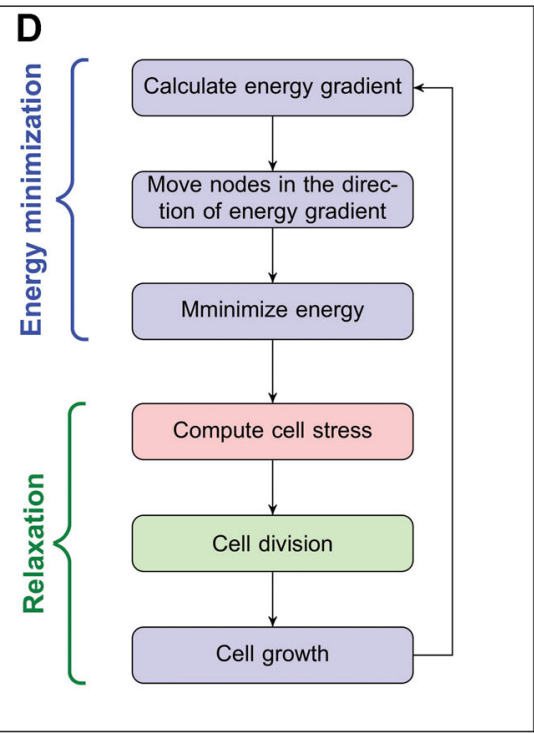

Figure 1. Schematic diagram of model

(A) Plant tissue structure. From the outside to the inside are the outer cell wall, epidermal cells, and inner cells. (B) Plant tissue is simplified into a vertex model. Epidermal cells and inner cells are represented by polygons. The stiffness of outer cell walls was set greater than that of inner cell walls, which was reflected by different wall thicknesses. (C) Schematic for polarized cell growth via cellular stress. A cell sustains mechanical stresses derived from the turgor pressure of its inside (outward light green arrows) as well as neighboring cells (inward light green arrows). The cell growth is promoted in the direction orthogonal to the maximal stress. When the cell volume (cell area in $2 \mathrm{D}$ ) reaches a threshold, cell division occurs along the direction of maximal stress. Calculated maximal tensile stress are shown as red arrows with the length proportional to the intensity of stress. The differential cell growth in different directions are shown as blue arrows. The orientation of cell division is shown as a red dotted line. (D) Modeling framework for numerical simulation.

(insets in Figure 2A, D, and $G$ ). We first started simulations with an elliptical organ with an aspect ratio of 1.2 (Figure $2 \mathrm{~A}-\mathrm{C}$, initial shape shown in the inset). With proper epidermal restriction, as seen in normal plants (Kutschera and Niklas 2007), the organ shape was smooth and elongated after growth (Figure $2 \mathrm{~A}$ ). The outermost inner cells tended to divide anticlinally, in which the division planes (white outlines) are perpendicular to the epidermal layer and organ surface. A partial reduction in epidermal restriction through the removal of the outer cell wall of the epidermal layer (Figure 2B) eliminated mechanical resistance and led to an organ with a twisted boundary. Nevertheless, the majority of cell division planes (white outlines in Figure 2B) of the outermost inner cells were still perpendicular to the epidermal layer; this outcome may reflect tensile force from the epidermal cells dictating the direction of cellular growth, such that cells preferentially grow and divide perpendicularly. Finally, the entire epidermal layer was removed to fully abolish the epidermal restriction (Figure $2 \mathrm{C}$ ). In this case, the orientations of the division planes in several outer layers of inner cells were significantly altered which is parallel to the organ surface, although the organ shape was less twisted. The final shape and division pattern are consistent with the in planta observations (Figure $5 \mathrm{~K}, \mathrm{~L}$ ). Thus, with no epidermis, the direction of maximum tensile stress of the outermost cells may change, which leads to further alterations in the cell division orientation from anticlinal to periclinal. Moreover, the organ shape becomes even less anisotropic than its initial state with the abolishment of entire epidermis (Figure 2C), indicating that the epidermal restriction is required for anisotropic organ shape formation. Together, our simulation results show the importance of epidermal restriction on organ shape stability and anisotropy.

We further analyzed additional initial organ shapes, including a less anisotropic organ shape with an initial aspect ratio of 1.1 (Figure 2D-F). Similar trends were observed with proper and completely eliminated epidermal 

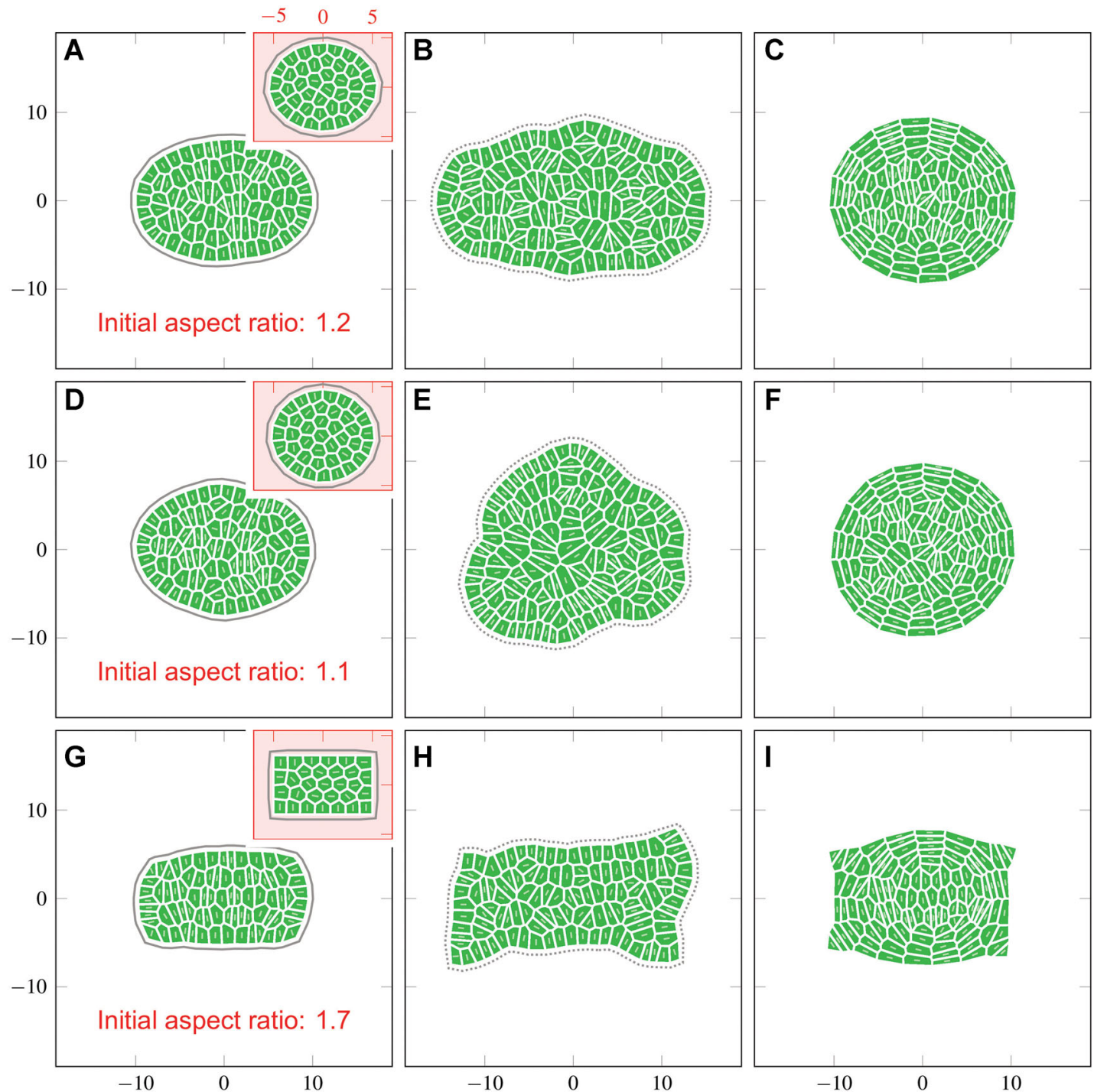

Figure 2. Effects of different types of epidermal constraints on plant tissue development

The three rows show simulations starting from two ellipses with different oval contours (A, D) and a rectangle (G), and each column represents a different type of epidermal constraint. Column I (A, D, G) represent a normal epidermal layer, depicted as a solid line, such that the outermost (epidermal) cells are subjected to tensile forces from the outer cell wall. Column II (B, E, H) represents a partially eliminated epidermal layer, depicted as a dotted line; there is no outer cell wall, but the outermost (epidermal) cells are forced to peripherally grow and periclinal divide. In column III (C, F, I), the epidermal layer is completely eliminated; there is no outer cell wall, and the outermost cells (which perhaps cannot be called epidermal cells) grow and divide in the same manner as inner cells.

restriction (Figure 2D, F). However, partially eliminated epidermal layers (Figure $2 \mathrm{E}$ ) led to the final shape becoming less anisotropic than its initial state (Figure 2D), indicating that the proper epidermal restriction is required for anisotropic organ shape formation, especially the shape with a small initial aspect ratio.

To examine whether the epidermis can smooth out extreme protrusions of the plant surface, we computed organ development starting from initial shape of a rectangular with an aspect ratio of 1.7. As shown in Figure $2 \mathrm{G}$, despite the strong anisotropy, mechanical restriction evened out the shape angles, resulting in a smoothly developed organ. In contrast, partial removal (Figure $2 \mathrm{H}$ ) or complete removal (Figure $2 \mathrm{l}$ ) of the epidermal restriction led to a grown organ with angles, suggesting reduced robustness in shape formation. 


\section{Epidermal constraints eliminate growth noise}

The above simulation results highlight that organ shape robustness requires epidermal restriction. To test this relationship more directly, we introduced cell growth perturbations, which are an inherent feature of real cells. To mimic cell growth stochasticity, we started with an elliptical shape of the same aspect ratio but randomly divided the inner tissues into the same number of cells in each independent replicate (Figure 3).

With proper epidermal restriction, we found that the final shape was insensitive to initial variations in the distribution and shape of cells, resulting in highly robust final shapes (column I in Figure 3). When the epidermal restriction was partially removed (column II in Figure 3), the final shapes could be highly variable (compare the values of shape variability between Figure $3 \mathrm{G}$ and $\mathrm{H}$ ). Thus, the cell growth stochasticity can be amplified without sufficient epidermal restriction. On the other hand, when the epidermal restriction was fully removed (column III in Figure 3), the final shapes lost the original shape polarity and degenerated into circles. Collectively, the
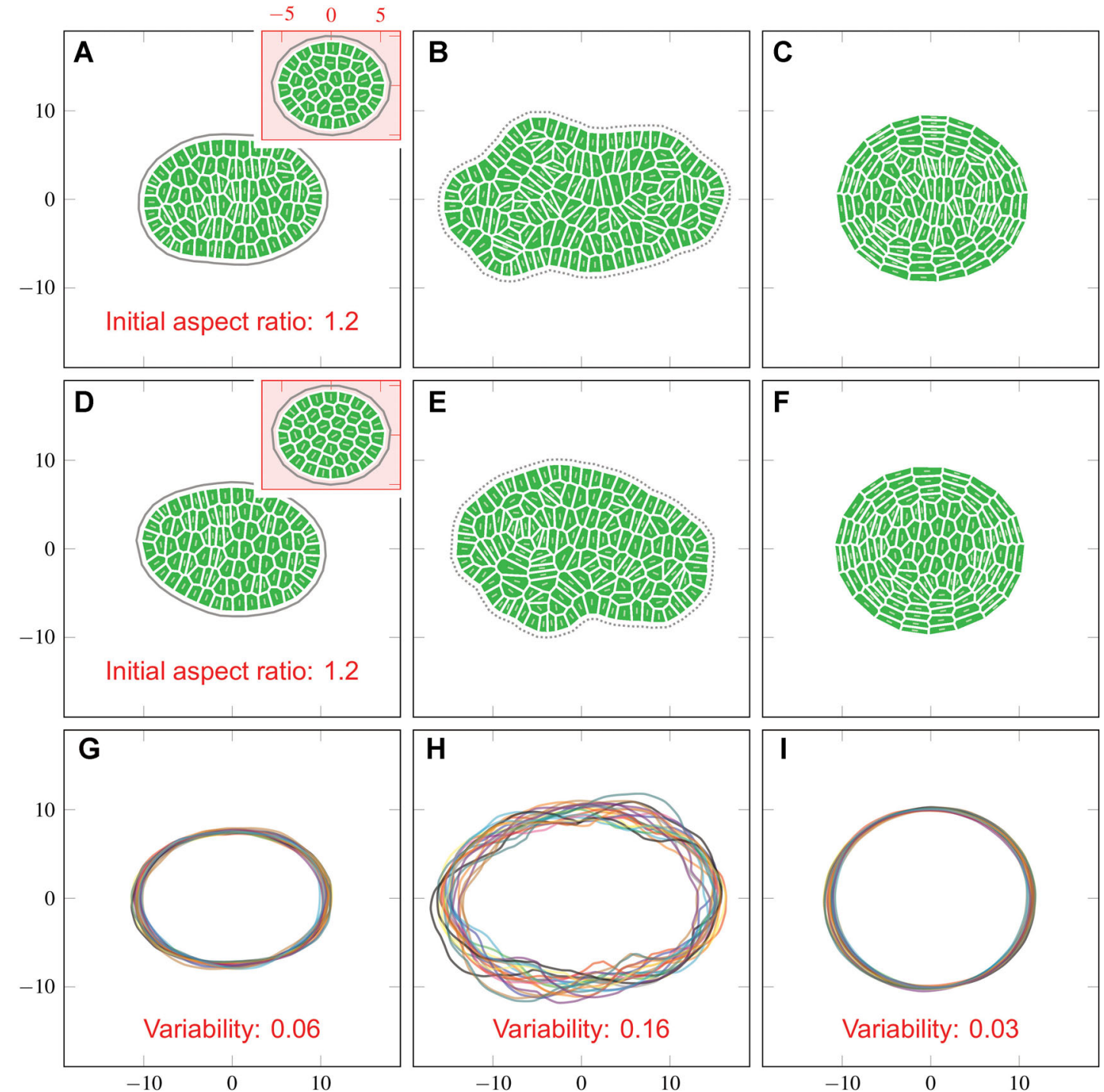

Figure 3. Role of epidermal constraints in eliminating the growth noise generated by the inner tissue In the first (A-C) and second (D-F) rows, the simulations start with an initial ellipse tissue with the same contour and cell number but slight differences in the distribution and shape of cells between each row. Each column illustrates a different type of epidermal constraint (as described in Figure 2). The third row show superimposed outlines of organ shapes from multiple simulations with full $(\mathbf{G})$, partial $(\mathbf{H})$ or no $(\mathbf{I})$ epidermal restriction. 
simulation results strongly support the ability of the epidermal layer to normalize internal tissue growth randomness, leading to both reproducible and anisotropic organ shapes.

\section{Reduced organ shape robustness in atml1 pdf 2 plants}

To experimentally test the predicted roles of the epidermal layer on organ morphogenesis, we used atml1 pdf2 double mutant plants (Abe et al. 2003). Both ATML1 (Arabidopsis thaliana MERISTEM LAYER 1) and PDF2 (PROTODERMAL FACTOR 2) are specifically expressed in the epidermal cell layer and redundantly define the epidermal fate. By scanning electron microscopy (SEM), we observed that the epidermal cells do not properly develop in atml 1 pdf2 mutant leaves (Figure $4 \mathrm{~A}-\mathrm{C}$ ). The pavement cells lose the jigsaw puzzle-like shapes which is typical in the epidermis of wild-type leaves (Figure 4B, left), and become into long rectangle shapes (Figure $4 \mathrm{~B}$, right). Moreover, the adhesion of neighboring pavement cells is largely compromised in atml 1 pdf2 and results in many cracks throughout the entire leaf (Figure $4 \mathrm{~B}, \mathrm{C}$ ), indicating that the epidermal restriction is reduced to the level not sufficient to resist inner-tissue growth. This cell adhesion defect in atml1 pdf2 epidermis is consistent with the fact that ATML1 is required for cuticle formation and outer cell wall integrity maintenance (Ingram and Nawrath 2017). Thus, the mechanical properties of epidermis are likely to be altered in atml1 pdf2 leaves.

To examine the mechanical properties of epidermal layers in wild-type and mutant leaves, we performed atomic force microscopy (AFM) to directly measure the elastic modulus of the outer walls in epidermal cells. Atomic force microscopy-based micromechanical approaches have been demonstrated as important assessments to the mechanical properties of plant tissue at a cellular resolution (Milani et al. 2013; Routier-Kierzkowska and Smith 2013; Vogler et al. 2015). It was revealed that the elastic modulus is dramatically reduced in the pavement cells of atml 1 $p d f 2$ leaves compared with the one in the wild type, reflecting a decrease in outer cell wall stiffness (Figure 4D-F). Based on the morphology and
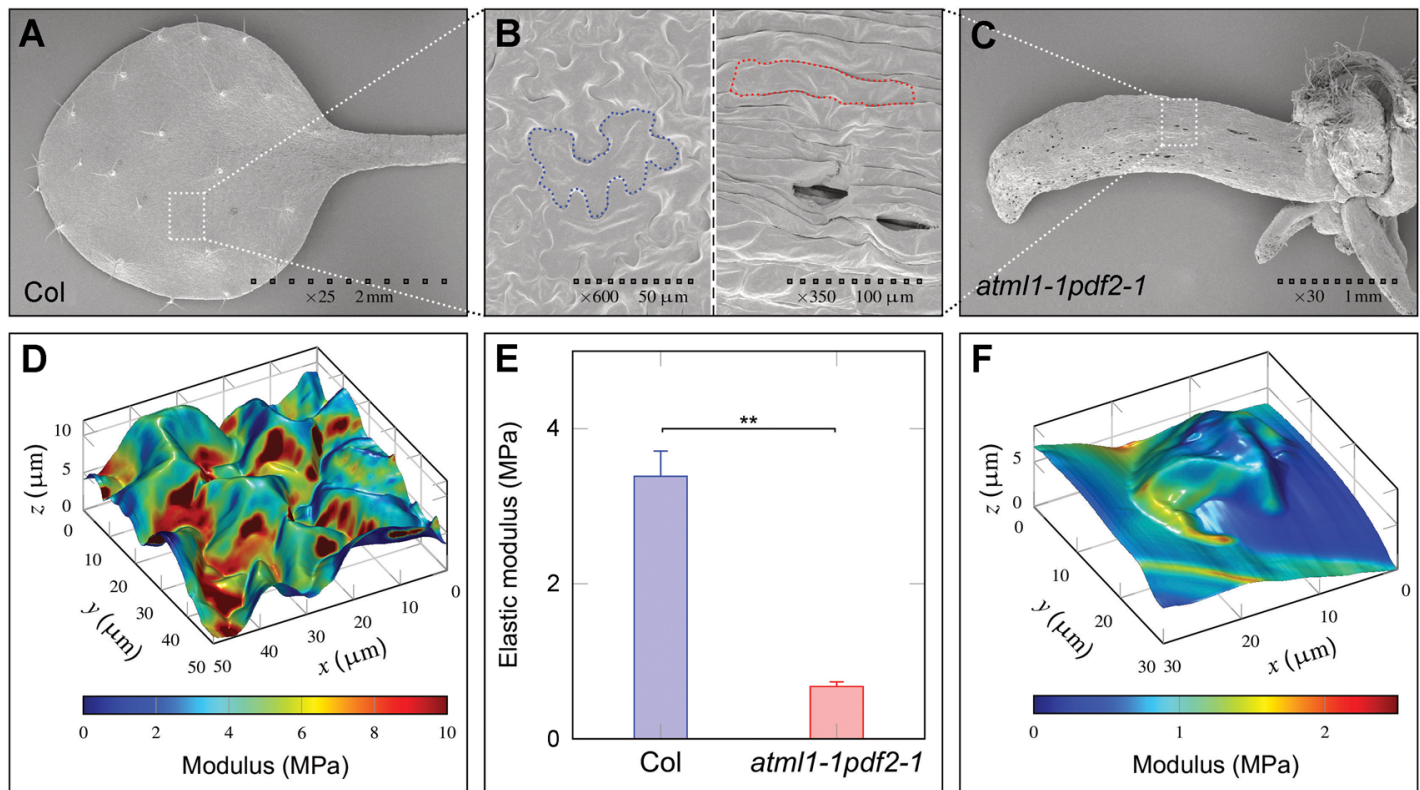

Figure 4. atml1 pdf2 double mutant displays reduced epidermal restriction and stiffness in leaves

(A-C) SEM images of adaxial leaf phenotypes in $\mathrm{Col}(\mathbf{A})$ and atml1-1 pdf2-1 (C). The morphology of leaf pavement cells of Col (highlighted by blue dashed line) and atml1-1 pdf2-1 (highlighted by red dashed line) are shown in (B). Note that the leaf epidermis of atml1-1 pdf2-1 is distributed by cracks from pavement cell separation (B, right). Scale bars are indicated as dotted lines. (D-F) Three-dimensional rendering of adaxial leaf epidermal cell topography overlaid by elasticity obtained by AFM in Col (D) and atml1-1 pdf2-1 (F). Note that different scales of elastic modulus are used. Quantification of elastic modulus of epidermis obtained by AFM in Col $(n=6$ leaves from 6 individual plants) and atml1-1 pdf2-1 $(n=7$ leaves from 7 individual plants) leaves are shown in $(E)$. Values are mean $\pm S D$. ${ }^{* *} P<0.01$ by student's $t$-test. 
mechanical defects, atml1 pdf2 mutant provides an ideal model system with a reduced epidermal constraint to test our simulation results.

Consistent with previous reports, we observed widespread developmental defects in atml1 pdf2 shoot organs, with many abnormal and irregularly shaped organs. Furthermore, the abnormal organ shapes were highly variable (Figures $5 \mathrm{~A}-\mathrm{C}, \mathrm{S} 1 \mathrm{~A}-\mathrm{F}$ ). We sectioned wild-type and atml1 pdf2 mature leaves to analyze the variations in leaf shape. Whereas wild-type leaves had a consistently flattened laminar shape (Figure 5D), atml1 pdf2 leaves were often substantially thicker and of variable shapes with disordered cell organization (Figure 5E, F). Quantification of shape variability in multiple wild-type and mutant leaves further indicated that the epidermal constraints confer the organ shape robustness in both transverse and horizontal directions (Figures $5 \mathrm{G}-\mathrm{I}, \mathrm{S} 1 \mathrm{G}-\mathrm{I}$ ). Since the patterns of leaf polarity genes do not change in atml1 pdf2 leaves (Ogawa et al. 2015), we reasoned that the leaf phenotypes are largely due to the alteration in epidermal mechanical properties.

To analyze cell division patterns, we combined physical and optical tissue sectioning with FB28 staining (for cellulose-containing structures) to identify new cell walls derived from recent cell divisions (Figure S2). By analyzing cells from different sections along the proximal-distal axis in the fourth youngest leaves $\left(\mathrm{P}_{4}\right)$, we found that most inner cell divisions were perpendicular to the mediolateral axis in wild-type leaves (Figure $5 \mathrm{~J}$ ). On the other hand, epidermal cell division was always perpendicular to the outer surface in wild-type leaves (Figure $5 \mathrm{~J}$ ). The cell division patterns were substantially different and more randomized in atml $p d f 2$ leaves, with more cell divisions parallel to the organ surface in both the outermost layer (supposed epidermis in mutants) and inner tissue (Figure 5K, L). Taken together, atml1 pdf2 plants are unable to maintain robust and anisotropic leaf growth, and the highly variable organ shapes with randomized cell division patterns in these plants are consistent with the simulation results.

\section{Effect of heterogeneous epidermal tensile resistance on organ shape development}

Previous atomic force microscopy measurements showed that the Young's modulus, which describes

www.jipb.net tensile elasticity, of the epidermal walls can undergo spatiotemporal changes (Peaucelle et al. 2011, 2015; Qi et al. 2017). It is generally assumed that differences in elasticity correlate with wall extensibility that affects cell expansion and organ growth. Tensile elasticity is measured by tensile resistance strength in our model. We therefore tested if variations in epidermal wall tensile resistance strength regulate organ shapes.

To examine how a heterogeneous epidermal resistance strength affecting an initial isotropic shape, we first simulated a circular organ with differences in epidermal tensile resistance strength. Specifically, if half of the epidermal layer had lower tensile resistance strength (soft) than the other half (stiff), the resulting organs always exhibited greater expansion on the soft side, leading to an anisotropic growth (Figure $6 \mathrm{~A}-\mathrm{C}$ ). This growth pattern is independent of the relative locations of the soft and stiff parts in the epidermal layer. We next examined how a heterogeneous epidermal tensile resistance affecting an initial anisotropic shape (Figure 6D-F). We also introduced low anisotropy and started with an initial ellipse with an aspect ratio of 1.1. When the soft region spanned one-quarter of the epidermal wall, the final organ had a gourd-like shape (Figure 6D). When the epidermal wall had alternating soft and stiff regions (Figure 6E inset), the anisotropy of the final shape was more pronounced (Figure 6E). Finally, when one-quarter of the epidermal wall had higher stiffness, the final organ had a bottom convex and top concave shape (Figure 6F), which was similar to a natural flattened leaf as shown in Figure $5 \mathrm{~J}$. Therefore, the heterogeneity in tensile resistance of the epidermal walls may provide the source of organ shape variety.

\section{Sensitivity to parameters}

In order to analyze model robustness, we performed sensitivity analysis on parameters. We changed the parameters individually, re-simulated, and checked if and how these changes would affect the final shapes. Other parameters were based on values given in Section Implementation. The effect of a $10 \%$ increase or $10 \%$ decrease of parameters (including turgor pressure $p$, wall spring constant $k_{w}$, cell area threshold $A_{\text {div }}$ and cell growth rate $r$ ) on the variability of shapes is shown in Figure S3. Neither a 10\% increase nor 10\%

December 2020 | Volume 62 | Issue 12 | 1853-1867 

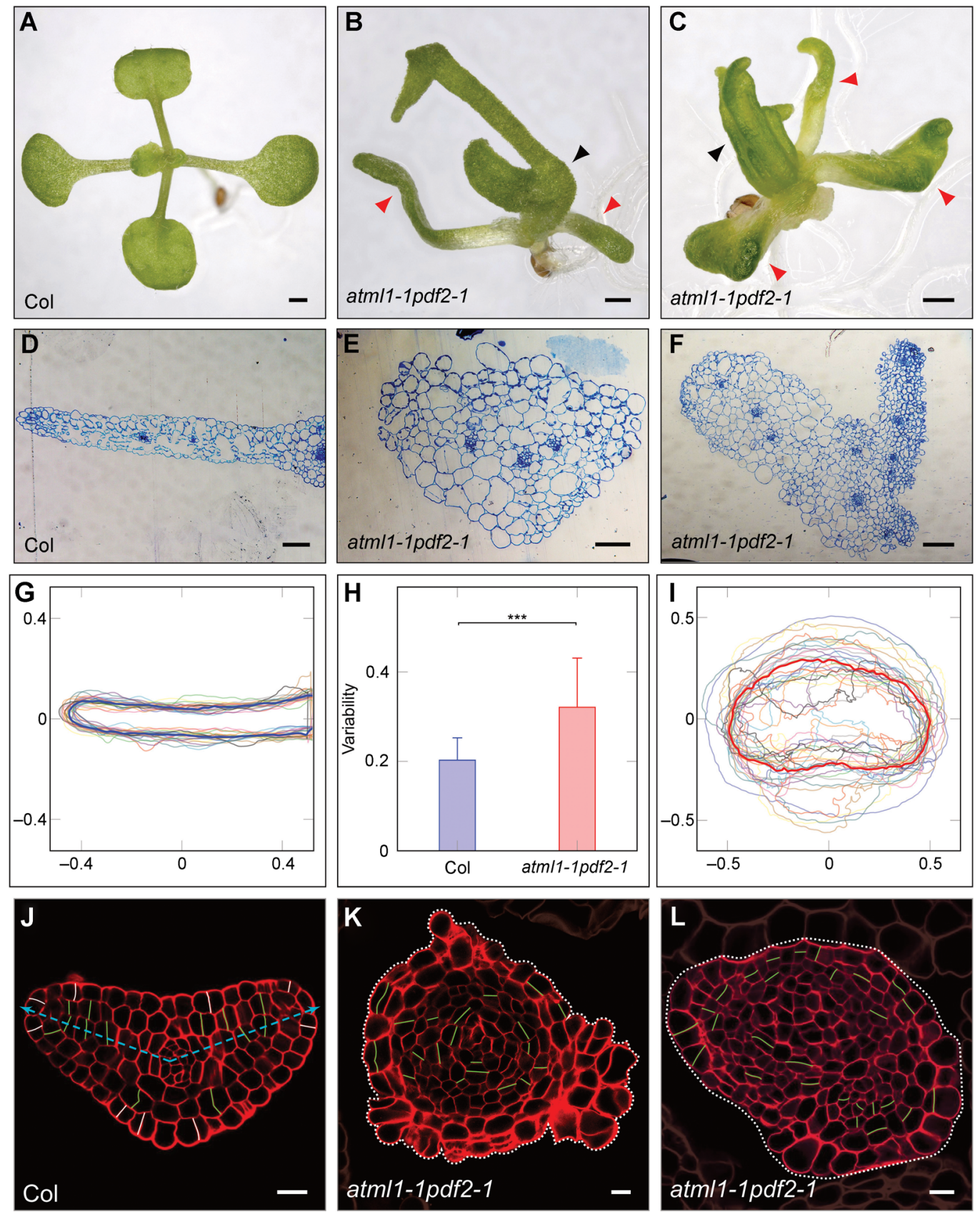

Figure 5. Defective mechanical properties of epidermis lead to reduced leaf shape robustness

(A-C) Seedling phenotypes of 15-d-old Col with normal flattened cotyledons and true leaves (A) and atml1-1 pdf2-1 mutants with rod-like (red arrowheads) and fused (black arrowheads) leaves due to compromised epidermis development (B-C). (D-F) Semi-thin transverse sections showing the cellular organization in Col (D) and atml1-1 pdf2-1 true leaves (E, F). Note that dramatic organ deformations occur in atml1-1 pdf2-1 leaves. (G-I) Superimposed outlines of multiple individual true leaves from $\mathrm{Col}(\mathrm{G})$ and atml1-1 pdf2-1 (I), showing the shape variability in the transverse direction. Due to the bilateral symmetry nature of Col leaf, only the half outline of each leaf sample is shown. The median outlines of leaf shapes are represented as blue and red line for Col and atml1-1 pdf2-1, respectively. Quantification of leaf shape variability in Col and atml1-1 pdf2-1 is shown in $(\mathrm{H})$. Values are mean \pm SD. $* * * P<0.001$ by student's $t$-test. $n=23$ for Col, $n=26$ for atml1-1 pdf2-1. (J) Cell division pattern in Col leaf primordium. The cell division planes are labeled white in epidermal cells and green in inner cells. The medio-lateral axes of the leaf primordium are shown by the dashed lines/ arrows in blue. (K) Cell division pattern in atml1-1 pdf2-1 leaf primordium with a rod-like form. The cell division planes are labeled green in all cells due to indistinguishable epidermal and inner cell identities. (L) Cell division pattern in atml1-1 pdf2-1 leaf primordium fused by several rod-like organs. Scale bars: $500 \mu \mathrm{m}$ in (A-C), $50 \mu \mathrm{m}$ in (G-I), and $10 \mu \mathrm{m}$ in (J-L). 

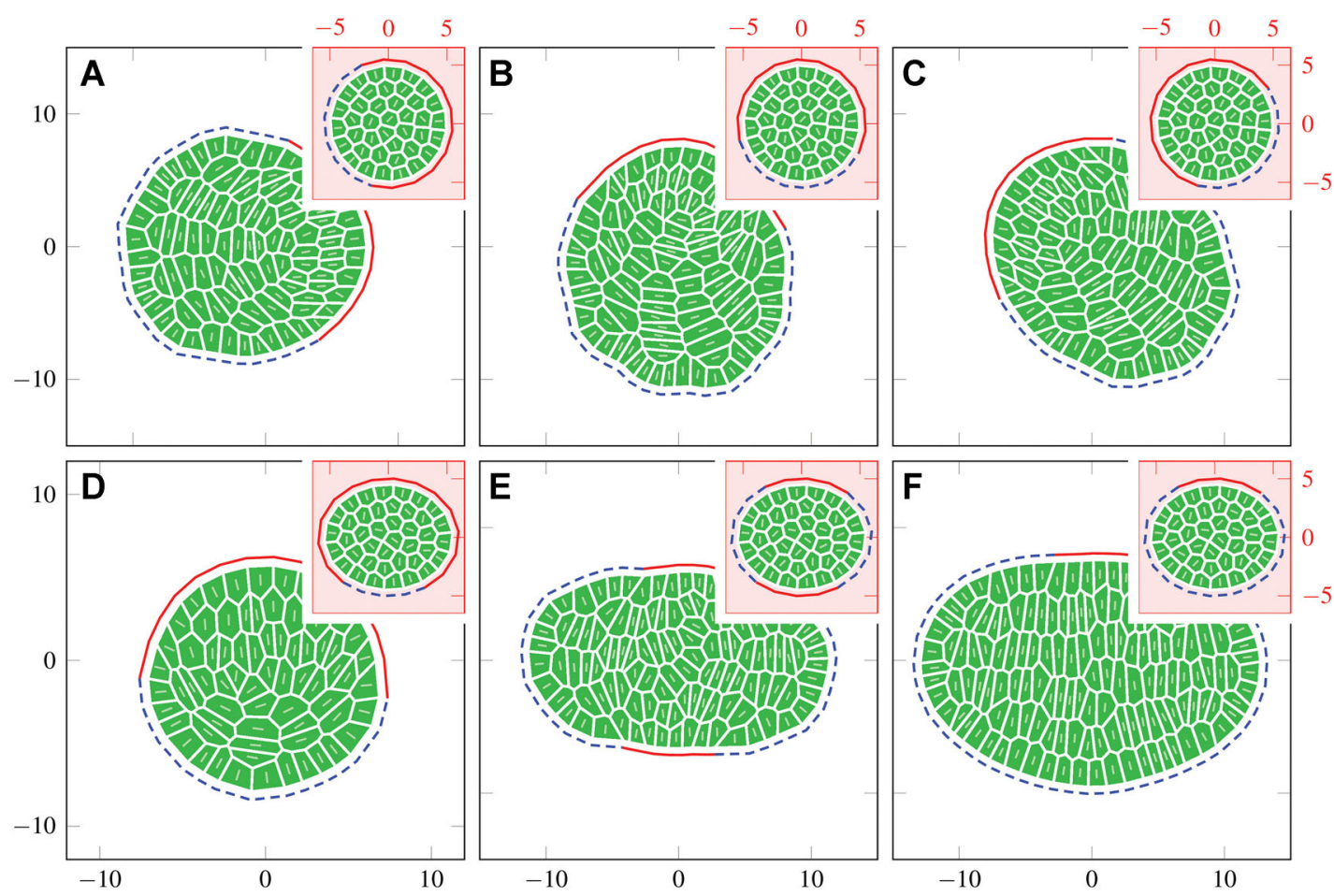

Figure 6. Organ shape variability with heterogenous epidermal tensile resistance strength (A-C) In each example, the initial pattern comprises two approximately equal soft and stiff cell wall segments around a circle. The orientation of the soft and stiff segments determines the preference of tissue growth. (D-F) The initial patterns comprise two or four unequal soft and stiff cell wall segments around an ellipse. The different patterns of epidermal elasticity result in different developed shapes. Blue dashed lines represent an outer cell wall with low tensile resistance strength (soft), and red solid lines represent an outer cell wall with high tensile resistance strength (stiff) $\left(k_{\text {red }}^{\top}=2 k_{\text {blue }}^{\top}\right)$.

decrease of all parameters led to a substantial deviation of the variability value, suggesting that the small variation of parameters does not affect the conclusion of this paper.

\section{DISCUSSION}

Organ shape development is highly robust despite variations in the levels of different molecules, such as transcripts or proteins, in cells. While there has been increasing focus on stochasticity and heterogeneity, especially their roles in patterning, fairly little is known about how shape robustness is achieved with these variabilities. The present study takes advantage of recent developments in mechanical modeling approaches to investigate the impact of restriction by the tensile epidermal layer on organ shape robustness.

\section{"Tensile skin" to maintain shape and to average noisy cellular growth}

Although cells are subunits in multicellular plant forms, the existence of epidermis as the outermost cell layer highly coordinates individual cell activities, making the organism as a whole during morphogenesis. (Kaplan and Hagemann 1991; Kaplan 1992) The "tensile skin" theory proposes that the epidermis is a driving force of organ growth rate (Kutschera and Niklas 2007). However, it is unclear whether the epidermis contributes to aspects of organ patterning other than size. In this study, we show that the epidermis is essential for organ shape maintenance. Without the sufficient epidermal restriction, organ shape is highly variable and more isotropic, as observed in atml1 pdf2 mutant plants (Figures 5, S1). Our computational modeling results indicated that this regulation by the epidermis is achieved at two levels. 
First, proper epidermal restriction allows the maintenance of existing organ shape asymmetry (Figure 2). Without sufficient epidermal restriction, an organ would exhibit isotropic growth, leading to a loss of asymmetry or a reduced anisotropic level. Our modeling results also showed that in terms of isotropy, an optimal strength of epidermal restriction is essential for shape asymmetry maintenance (Figure S4). With an increased level of epidermal restriction, an organ may also exhibit isotropic growth accompanied by reduced growth rate, as the "tensile skin" theory would predict.

Second, epidermal restriction averages variations in cell growth, which is a widespread phenomenon given that virtually every gene has stochastic expression. By introducing low levels of randomness in cell growth, the computational modeling showed that without epidermal restriction, stochasticity would lead to large shape variations.

As cell growth represents a balance between epidermal restriction and internal turgor pressure, these two factors must be coordinated. Because the measurement of turgor pressure remains technically challenging in most cell types, we assumed a uniform turgor pressure in our models. When variations in turgor pressure exist, as recently proposed (Robinson and Kuhlemeier 2018), turgor difference may coordinate with the epidermal layer to ensure organ shape robustness.

The epidermis not only provides mechanical restriction to inner tissues, but also serves as a source of biochemical signals that directs development (Chickarmane et al. 2012; Knauer et al. 2013; Gruel et al. 2016; Han et al. 2020). ATML1 and PDF2 are key regulators of epidermal cell differentiation, and the atml1 pdf2 mutants are expected to lose, at least partially, both mechanical restriction and epidermisderived biochemical signals (Abe et al. 2001). In this study, we were able to explain the observed organ shape variation of atml1 pdf2 mutant plants by using only the reduced mechanical restriction of epidermis. The combination of theoretical and experimental analyses showed that a reduction of mechanical restriction alone is sufficient to substantially reduce organ shape robustness. Nevertheless, we do not exclude unidentified contributions from biochemical signals on organ shape robustness. Notably, Arabidopsis adaxial/abaxial patterning mutants also exhibit reduced organ shape asymmetry as in atml1 pdf2 (McConnell et al. 2001; Emery et al. 2003). However, the expression patterns of adaxial/abaxial identity genes, like PHB and FIL, remain unchanged in atml1 pdf2 leaves (Ogawa et al. 2015). This may indicate that the variable leaf shapes in atml1 pdf2 are not due to altered leaf polarity, which is different from adaxial/ abaxial patterning mutants.

\section{Varying mechanical properties of the epidermis may explain organ shape asymmetry establishment}

The development of functional organs requires a certain level of asymmetry accompanied by cell specification for specialized biological functions. Moreover, organ and tissue shaping requires gene regulators and cellular effectors. Upstream gene regulators, such as transcription factors, signal transduction components, and hormones, divide an organ into domains to form the initial patterns. Downstream cellular effectors translate fate decisions into physical shapes. Essential cellular machinery components are often utilized for this purpose and can lead to mechanical forces, as previously proposed (Keller 2012).

Extensive studies over the years have identified a large number of upstream gene regulators. Among others, we have identified initial morphogens for embryogenesis asymmetry breaking and leaf asymmetry patterning (Ueda and Laux 2012; Du et al. 2018). On the other hand, we know little about how patterns of morphogens can be translated into physical shape asymmetry. Our simulation showed that an epidermis with uneven mechanical properties could induce anisotropic tissue growth to establish organ asymmetry (Figure 6). In general, inner cells are less constrained by softer epidermal regions, allowing greater tissue deformation. Conversely, stiffer epidermal regions have a stronger restrictive effect on inner cell growth. Tissuescale mechanics could therefore increase the complexity of growth anisotropy. Nevertheless, heterogeneous mechanical properties can lead to heterogeneous growth, which is clearly an essential factor for physical shaping (Ali et al. 2014; Sampathkumar et al. 2014; Hamant 2017). During Arabidopsis and tomato leaf formation, a tissuesscale mechanical heterogeneity of cell walls, mediated by methyl-esterification modifications of pectin, is critical to the anisotropic growth and organ shape asymmetry (Qi et al. 2017). 


\section{METHODS}

\section{Hamiltonian}

We defined the system's Hamiltonian $H$ by summing up Hamiltonian $\mathrm{H}_{c}$ of $\mathrm{N}$ individual cells making up the 2D shape

$H=\sum_{c=1}^{N} H_{c}$

Cell Hamiltonians were used to describe the different energy compartments in the system, that is, turgor pressure and cell wall elastic deformation. For cell $c$, the potential energy was described by the following Hamiltonian:

$H_{c}=-p A_{c}+\frac{1}{2} \sum_{i=1}^{n_{c}} k_{c, i} L_{c, i}\left(\frac{\ell_{c, i}}{L_{c, i}}-1\right)^{2}$

where $p$ is turgor pressure, $A_{c}$ is the area of cell $c$. $k_{c, i}=1 / 2 k_{w}$ for a segment of inner cell walls (the factor $1 / 2$ comes from the fact that inner walls are shared by two contiguous cells in our vertex model) and $k_{c, i}=3 k_{w}$ for a segment of outer cell walls of epidermal cells. $k_{w}$ defines the spring constant of the inner cell wall per unit length. Note that with this convention the outer epidermal cell walls are threetimes stiffer than inner walls (shared by two cells). $L_{c, i}$, $i=1,2, \cdots, n_{c}$, is the rest length of each wall segment of cell $c, \ell_{c, i}$ is the actual length of cell wall segment $i$. The first term on the right side of the above equation accounts for the driving force of growth derived from turgor pressure. The second term represents elastic energy in cell walls.

The total energy of the system is a function of the coordinates of all vertices, thus the forces applied to vertex $k$ can be derived from the following formula

$f_{k}=\left(f_{x_{k}}, f_{y_{k}}\right)=-\frac{\partial H}{\partial r_{k}}$

where $r_{k}=\left(x_{k}, y_{k}\right)$ is the vector of coordinates of vertex $k$.

\section{Stress of a cell}

A cell is subjected to local stress exerted by surrounding cells. The following equations define stress tensor $\mathbf{S}_{i}$ on cell $i$ by calculating the force at every vertex as the energy's gradient $f_{k}=-\nabla_{k} H_{i}$ and www.jipb.net interpolating the force at two vertices linearly along the edge between them (Alim et al. 2012):

$$
\begin{aligned}
S_{c, x x}= & \sum_{i=1}^{n_{c}}\left(\frac{f_{x_{i}} x_{i}+f_{x_{i+1}} x_{i+1}}{3 A_{c}}+\frac{f_{x_{i}} x_{i+1}+f_{x_{i+1}} x_{i}}{6 A_{c}}\right) \\
S_{c, y y}= & \sum_{i=1}^{n_{c}}\left(\frac{f_{y_{i}} y_{i}+f_{y_{i+1}} y_{i+1}}{3 A_{c}}+\frac{f_{y_{i}} y_{i+1}+f_{y_{i+1}} y_{i}}{6 A_{c}}\right) \\
S_{c, x y}= & \sum_{i=1}^{n_{c}}\left(\frac{f_{x_{i}} y_{i}+f_{y_{i}} x_{i}+f_{x_{i+1}} y_{i+1}++f_{y_{i+1}} x_{i+1}}{6 A_{c}}\right. \\
& \left.+\frac{f_{x_{i}} y_{i+1}+f_{y_{i}} x_{i+1}+f_{x_{i+1}} y_{i}++f_{y_{i+1}} x_{i}}{12 A_{c}}\right)
\end{aligned}
$$

where $x$ and $y$ are a cell's vertex coordinates, and $A_{c}$ denotes the cell's area. The direction $\boldsymbol{e}$ (unit vector) that maximizes the stress can be defined as

$\boldsymbol{e}^{t} \mathbf{S}_{c} \boldsymbol{e}=\max \left\{\mathbf{u}^{t} \mathbf{S}_{c} \mathbf{u}_{\|\| \|=1}\right.$

\section{Mechanical equilibrium}

Cell positions and shapes of a leaf are determined by the mechanical equilibrium of all vertices. This is achieved by minimizing the total energy of all cells in the system:

$\min _{\left\{r_{k}\right\}} H\left(\left\{r_{k}\right\}\right)$

where $H$ is defined by Equations (1) and (2). $\left\{\boldsymbol{r}_{\mathrm{k}}\right\}$ represents the set containing all vertices.

\section{Modeling cell growth}

In our model, cell growth is achieved by increasing the natural lengths of the wall springs associated with the growing cell, simulating biosynthesis of new wall materials and plastic wall deformation (Rudge and Haseloff 2005):

$\frac{d L_{j}}{d t}=\lambda L_{j}, \lambda=r[1-\eta(\hat{\mathbf{s}} \cdot \hat{\imath})]$,

where $r$ is the cell growth rate, $\hat{\mathbf{s}}=\left(\cos \theta_{p}, \sin \theta_{p}\right)$ is the unit direction of maximum stress of the cell, and $\hat{\imath}$ is the unit direction of the walls. $\eta \in[0,1]$ represents the strength of the stress feedback on cell growth and division orientations. With $\eta=0$, there is no stress feedback and cells exhibit isotropic growth. With $\eta=1$, walls aligned closely perpendicular to the direction of maximum tensile stress $\hat{s}$ exhibit mostly growth.

December 2020 | Volume 62 | Issue 12 | 1853-1867 


\section{Modeling cell division}

In our model, cell division is achieved by a straight line (new cell wall) going through the centroid of the cell. The new cell wall aligns in the direction of the maximum stress of the cell.

\section{Implementation}

The initial shape input to the model was usually a Voronoi diagram. Each polygon represents a cell. Typically, the Voronoi diagram was generated by randomly and uniformly distributed points within a specific boundary. During simulations, we used the following parameters (in arbitrary units): turgor pressure $p=1$, wall spring constant $k_{w}=10$, growth constant $\lambda=0.5$, and cell area threshold $A_{\text {div }}=2.75$. All simulations performed seven time steps. The energy was minimized using the largescale algorithm, implemented by the fmincon function provided by the MATLAB Optimization Toolbox.

\section{Plant materials and growth conditions}

Arabidopsis thaliana ecotype Col-o plants were germinated on Murashige and Skoog agar plates $(1 / 2 \times M S$ salts, $0.8 \%$ agar, $1 \%$ sucrose, $\mathrm{pH}=5.8)$ under $8 \mathrm{~h}$ light and $16 \mathrm{~h}$ darkness for $15 \mathrm{~d}$. The phenotypes of wild-type and mutant plants were observed using a Zeiss Discovery V20 stereomicroscope. The genotyping of the atml1-1 pdf2-1 mutant was performed as previously described (Ogawa et al. 2015) with a modified ML1-F1 primer to improve $P C R$ efficiency. The following gene-specific primers were used for amplification: ML1-F1 (5'-CATC ATCATATGTTCGATATGACGCCG-3') and ML1-3N (5'-GTT TTGGAGCTACAGGGATCCAGA-3') for ATML1 and PDF2-F1 (5'-GATCAGTGCCTTGAAGGAAA-3') and PDF2-R2 (5'-CTG TTGTCGACATTGTTGTC- $3^{\prime}$ ) for PDF1. The primers used for detecting T-DNA were ML1-F1 and JL202 (5'-CATTTTATAATAACGCTGCGGACATCTAC-3') for atml1-1 and PDF2-F1 and JL202 for pdf2-1.

\section{Atomic force microscopy}

The procedure of AFM was performed as previously described (Qi et al. 2017). For material preparation, leaves were detached from 15-d-old wild-type and atml1-1 pdf2-1 mutant seedlings. A small area without trichome in the middle part of the leaf was further dissected with a syringe tip and pasted onto a Petri dish. The measurement was carried out with the samples under water at room temperature. Data were analyzed with Nanoscope
Analysis software (version 1.8). We neglected outlier data obtained by AFM. Outliers are defined as elements more than two standard deviations from the mean.

\section{Scanning electron microscopy}

In material preparation for SEM, the oldest true leaf was collected from each 15-day-old wild-type seedling. As to atml1-1 pdf2-1 mutant in which the phyllotaxis is completely irregular, the largest true leaf in size was collected from each mutant plant. Leaves were fixed in pure methanol for $15 \mathrm{~min}$ and then dehydrated in 100\% ethanol for $30 \mathrm{~min}$ at room temperature. After one change of $100 \%$ ethanol, the tissues were stored in $100 \%$ ethanol overnight at room temperature. Tissues were dried with $\mathrm{CO}_{2}$ in a critical point drier and coated with gold in a sputter coater. Tissues were imaged using a Hitachi S-3000N variable pressure scanning electron microscope at an accelerating voltage of $5 \mathrm{kV}$.

\section{Agarose gel sectioning and microscopy}

Agarose gel sectioning was performed as previously described (Skopelitis et al. 2017) with minor modifications. Briefly, older Arabidopsis leaf primordia and cotyledons were removed, and the seedlings were collected into freshly prepared fixative solution (4\% paraformaldehyde and $0.015 \%$ Tween-20 in $1 \times \mathrm{PBS}, \mathrm{pH}=7.0$ ). Vacuum infiltration at $-0.075 \mathrm{MPa}(550 \mathrm{~mm} \mathrm{Hg})$ was performed twice for $10 \mathrm{~min}$ each time. Tissues were washed three times (10 min per wash) in $1 \times$ PBS and embedded into $6 \%$ low-melting agarose (Promega). Transverse sections $(40 \mu \mathrm{m})$ were obtained using a VT1000S vibratome (Leica).

To examine the cell division patterns, the sections were stained with $0.01 \%$ Fluorescent Brightener 28 (FB28) in 1X PBS for $20 \mathrm{~min}$ in darkness for cell wall labeling, followed by three washes ( $5 \mathrm{~min}$ per wash) in $1 \times$ PBS. Sections were mounted in $90 \%$ glycerol in $1 \times$ PBS and imaged using a Nikon A1 confocal laser scanning microscope. To detect FB28 staining, a $405 \mathrm{~nm}$ laser line was used for excitation, and emission was collected at 425$475 \mathrm{~nm}$.

\section{Semi-thin sectioning}

The oldest true leaves of Col-o and atml1-1 pdf2-1 were fixed in FAA solution under vacuum for $3 \times 10 \mathrm{~min}$ at $0.075 \mathrm{MPa}$ then maintained in fresh FAA solution overnight at $4^{\circ} \mathrm{C}$. After dehydration in an ethanol series to $100 \%$ ethanol, tissues were embedded using 
the SPI low viscosity Spurr's kit (SPI Supplies). Sections $(2 \mu \mathrm{m})$ were obtained using a Leica RM 2265 rotary microtome and stained with $1 \%$ toluidine blue in water supplemented with $1 \%$ sodium tetraborate at $65^{\circ} \mathrm{C}$ for $20-30 \mathrm{~min}$. Sections were mounted in $50 \%$ glycerol for optical microscopy with an Olympus BX60 microscope equipped with a Nikon DS-Ri1 camera.

\section{Leaf shape robustness analysis}

The first pair of true leaves were detached from each 15-d-old Col wild-type seedling. As to atml1-1 pdf2-1 mutant plant in which the phyllotaxis is completely irregular, the largest true leaf in size was chosen. For shape robustness analysis in the horizontal direction, leaves were laid flat and photographed using a Zeiss Discovery V20 stereomicroscope. For shape robustness analysis in the transverse direction, leaves were embedded in agarose gel followed by sectioning along the adaxial-abaxial axis. The sections were stained with FB28 and photographed using a Nikon A1 confocal laser scanning microscope. For the leaf shape recognition and measurement, we use matlab to binarize the image and get the contour of all the leaves. For the average shape of blade, we standardize all polygons (leaves shape) and then overlap them, and ensure that the centroids of polygons coincide and the major axes of ellipse fitted by polygons coincide. We take the boundary of the area region with more than half of the number of samples overlapped as the average shape. Shape variability (SV) of leaf $i$ from average shape is defined as follows:

$\mathrm{SV}_{i}=\frac{A_{i} \cup A_{\mathrm{ave}}-A_{i} \cap A_{\mathrm{ave}}}{A_{i} \cup A_{\mathrm{ave}}}$

where $A_{i}$ is the area enclosed by leaf $i$ outline, $A_{\text {ave }}$ is the area of average shape. Operators $U$ and $\cap$ represent Union and intersection respectively.

\section{ACKNOWLEDGEMENTS}

We thank Dr. Taku Takahashi for atml1-1 pdf2-1 seeds. The authors have no conflicts of interest to declare. This work was supported by National Natural Science Foundation of China (31825002, 31861130355, and 11972200); a Key Research Project of the Frontier Science of the Chinese Academy of Sciences Grant (ZDBS-LY-SM012) to Y.J.

\section{AUTHOR CONTRIBUTIONS}

Y.J., L.Z., and M.L. conceived the presented idea. L.Z., S.F., S.L., and M.L. designed the models. L.Z. and S.F. performed the numerical simulations. Y.J. and F.D. designed the experiments. F.D. and J.H. performed experiments. Y.J., S.F., F.D., and L.Z. wrote the paper. All authors discussed the results and contributed to the final manuscript.

\section{REFERENCES}

Abe M, Katsumata H, Komeda Y, Takahashi T (2003) Regulation of shoot epidermal cell differentiation by a pair of homeodomain proteins in Arabidopsis. Development 130: 635-643

Abe M, Takahashi T, Komeda Y (2001) Identification of a cisregulatory element for $\mathrm{L} 1$ layer-specific gene expression, which is targeted by an L1-specific homeodomain protein. Plant J 487-494

Ali O, Mirabet V, Godin C, Traas J (2014) Physical models of plant development. Annu Rev Cell Dev Biol 30: 59-78

Alim K, Hamant O, Boudaoud A (2012) Regulatory role of cell division rules on tissue growth heterogeneity. Front Plant Sci 3: 174

Araújo IS, Pietsch JM, Keizer EM, Greese B, Balkunde R, Fleck C, Hülskamp M (2017) Stochastic gene expression in Arabidopsis thaliana. Nat Commun 8: 2132

Bilsborough GD, Runions A, Barkoulas M, Jenkins HW, Hasson A, Galinha C, Laufs P, Hay A, Prusinkiewicz P, Tsiantis M (2011) Model for the regulation of Arabidopsis thaliana leaf margin development. Proc Natl Acad Sci USA 108: 3424-3429

Boudon F, Chopard J, Ali O, Gilles B, Hamant O, Boudaoud A, Traas J, Godin C (2015) A computational framework for 3D mechanical modeling of plant morphogenesis with cellular resolution. PLoS Comput Biol 11: e1003950

Chickarmane VS, Gordon SP, Tarr PT, Heisler MG, Meyerowitz EM (2012) Cytokinin signaling as a positional cue for patterning the apical-basal axis of the growing Arabidopsis shoot meristem. Proc Natl Acad Sci USA 109: 4002-4007

de Reuille PB, Bohn-Courseau I, Ljung K, Morin H, Carraro N, Godin C, Traas J (2006) Computer simulations reveal properties of the cell-cell signaling network at the shoot apex in Arabidopsis. Proc Natl Acad Sci USA 103: 1627-1632

Du F, Guan C, Jiao Y (2018) Molecular mechanisms of leaf morphogenesis. Mol Plant 11: 1117-1134

Emery JF, Floyd SK, Alvarez J, Eshed Y, Hawker NP, Izhaki A, Baum SF, Bowman JL (2003) Radial patterning of Arabidopsis shoots by class III HD-ZIP and KANADI genes. Curr Biol 13: 1768-1774

Galletti R, Verger S, Hamant O, Ingram GC (2016) Developing a 'thick skin': A paradoxical role for mechanical tension in maintaining epidermal integrity? Development 143: $3249-3258$

December 2020 | Volume 62 | Issue 12 | 1853-1867 
Gruel J, Landrein B, Tarr P, Schuster C, Refahi Y, Sampathkumar A, Hamant O, Meyerowitz EM, Jönsson H (2016) An epidermis-driven mechanism positions and scales stem cell niches in plants. Sci Adv e1500989

Hamant O (2017) Mechano-devo. Mech Dev 145: 2-9

Han H, Yan A, Li L, Zhu Y, Feng B, Liu X, Zhou Y (2020) A signal cascade originated from epidermis defines apicalbasal patterning of Arabidopsis shoot apical meristems. Nat Commun 11: 1214

Han Y, Chu X, Yu H, Ma YK, Wang XJ, Qian W, Jiao Y (2017) Single-cell transcriptome analysis reveals widespread monoallelic gene expression in individual rice mesophyll cells. Sci Bull 62: 1304-1314

Hervieux N, Tsugawa $S$, Fruleux A, Dumond $M$, RoutierKierzkowska AL, Komatsuzaki T, Boudaoud A, Larkin JC, Smith RS, Li CB, Hamant O (2017) Mechanical shielding of rapidly growing cells buffers growth heterogeneity and contributes to organ shape reproducibility. Curr Biol 27: e3464. 3468-3479

Hong L, Dumond M, Tsugawa S, Sapala A, Routier-Kierzkowska AL, Zhou $Y$, Chen $C$, Kiss $A$, Zhu $M$, Hamant $O$, Smith RS, Komatsuzaki T, Li CB, Boudaoud A, Roeder AH (2016) Variable cell growth yields reproducible organ development through spatiotemporal averaging. Dev Cell 38: 15-32

Hong L, Dumond M, Zhu M, Tsugawa S, Li CB, Boudaoud A, Hamant O, Roeder AHK (2018) Heterogeneity and robustness in plant morphogenesis: From cells to organs. Annu Rev Plant Biol 69: 469-495

Ingram G, Nawrath C (2017) The roles of the cuticle in plant development: Organ adhesions and beyond. J Exp Bot 68: $5307-5321$

Jönsson H, Heisler MG, Shapiro BE, Meyerowitz EM, Mjolsness E (2006) An auxin-driven polarized transport model for phyllotaxis. Proc Natl Acad Sci USA 103: 1633-1638

Kaplan DR (1992) The relationship of cells to organisms in plants: Problem and implications of an organismal perspective. Int J Plant Sci 153: S28-S37

Kaplan DR, Hagemann W (1991) The relationship of cell and organism in vascular plants: Are cells the building blocks of plant form? BioScience 41: 693-703

Keller R (2012) Physical biology returns to morphogenesis. Science 338: 201-203

Kierzkowski D, Nakayama N, Routier-Kierzkowska A, Weber A, Bayer E, Schorderet M, Reinhardt D, Kuhlemeier C, Smith R (2012) Elastic domains regulate growth and organogenesis in the plant shoot apical meristem. Science 335: 1096-1099

Knauer S, Holt AL, Rubio-Somoza I, Tucker EJ, Hinze A, Pisch M, Javelle M, Timmermans MC, Tucker MR, Laux T (2013) A protodermal miR394 signal defines a region of stem cell competence in the Arabidopsis shoot meristem. Dev Cell 24: 125-132

Kutschera U, Niklas KJ (2007) The epidermal-growth-control theory of stem elongation: An old and a new perspective. J Plant Physiol 164: 1395-1409

Lander AD (2011) Pattern, growth, and control. Cell 144: 955-969
Louveaux M, Julien JD, Mirabet V, Boudaoud A, Hamant $O$ (2016) Cell division plane orientation based on tensile stress in Arabidopsis thaliana. Proc Natl Acad Sci USA 113: E4294-4303

McConnell JR, Emery J, Eshed Y, Bao N, Bowman J, Barton MK (2001) Role of PHABULOSA and PHAVOLUTA in determining radial patterning in shoots. Nature 411: 709-713

Meyer HM, Teles J, Formosa-Jordan P, Refahi Y, San-Bento R, Ingram G, Jonsson H, Locke JC, Roeder AH (2017) Fluctuations of the transcription factor ATML1 generate the pattern of giant cells in the Arabidopsis sepal. elife 6: e19131

Milani P, Braybrook SA, Boudaoud A (2013) Shrinking the hammer: Micromechanical approaches to morphogenesis. J Exp Bot 64: 4651-4662

Ogawa E, Yamada Y, Sezaki N, Kosaka S, Kondo H, Kamata N, Abe M, Komeda Y, Takahashi T (2015) ATML1 and PDF2 play a redundant and essential role in Arabidopsis embryo development. Plant Cell Physiol 56: 1183-1192

Onoda Y, Schieving F, Anten NP (2015) A novel method of measuring leaf epidermis and mesophyll stiffness shows the ubiquitous nature of the sandwich structure of leaf laminas in broad-leaved angiosperm species. J Exp Bot 66: 2487-2499

Peaucelle A, Braybrook SA, Le Guillou L, Bron E, Kuhlemeier C, Hofte H (2011) Pectin-induced changes in cell wall mechanics underlie organ initiation in Arabidopsis. Curr Biol 21: 1720-1726

Peaucelle A, Wightman R, Hofte H (2015) The control of growth symmetry breaking in the Arabidopsis hypocotyl. Curr Biol 25: 1746-1752

Qi J, Wu B, Feng S, Lu S, Guan C, Zhang X, Qiu D, Hu Y, Zhou $Y$, Li C, Long M, Jiao $Y$ (2017) Mechanical regulation of organ asymmetry in leaves. Nat Plants 3: 724-733

Robinson S, Kuhlemeier C (2018) Global compression reorients cortical microtubules in Arabidopsis hypocotyl epidermis and promotes growth. Curr Biol 28: e1792. 1794-1802

Routier-Kierzkowska AL, Smith RS (2013) Measuring the mechanics of morphogenesis. Curr Opin Plant Biol 16: 25-32

Rudge T, Haseloff J (2005) A computational model of cellular morphogenesis in plants. In: Capcarrère MS, Freitas AA, Bentley PJ, Johnson CG, Timmis J, eds. Advances in Artificial Life. European conference on artificial life 2005. Springer Publishing, Berlin, Germany. pp. 78-87

Sampathkumar A, Yan A, Krupinski P, Meyerowitz EM (2014) Physical forces regulate plant development and morphogenesis. Curr Biol 24: R475-483

Savaldi-Goldstein S, Peto C, Chory J (2007) The epidermis both drives and restricts plant shoot growth. Nature 446: 199-202

Skopelitis DS, Benkovics AH, Husbands AY, Timmermans MCP (2017) Boundary formation through a direct threshold-based readout of mobile small RNA gradients. Dev Cell 43: 265-273

Smith RS, Guyomarc'h S, Mandel T, Reinhardt D, Kuhlemeier C, Prusinkiewicz P (2006) A plausible model of phyllotaxis. Proc Natl Acad Sci USA 103: 1301-1306

Ueda M, Laux T (2012) The origin of the plant body axis. Curr Opin Plant Biol 15: 578-584 
Uyttewaal M, Burian A, Alim K, Landrein B, Borowska-Wykret D, Dedieu A, Peaucelle A, Ludynia M, Traas J, Boudaoud A, Kwiatkowska D, Hamant O (2012) Mechanical stress acts via katanin to amplify differences in growth rate between adjacent cells in Arabidopsis. Cell 149: 439-451

Vogler H, Felekis D, Nelson BJ, Grossniklaus U (2015) Measuring the mechanical properties of plant cell walls. Plants (Basel) 4: 167-182

Wolf S, Mravec J, Greiner S, Mouille G, Hofte H (2012) Plant cell wall homeostasis is mediated by brassinosteroid feedback signaling. Curr Biol 22: 1732-1737

Zhao F, Du F, Oliveri H, Zhou L, Ali O, Chen W, Feng S, Wang Q, Lü S, Long M, Schneider R, Sampathkumar A, Godin C, Traas J, Jiao Y (2020) Microtubule-mediated wall anisotropy contributes to leaf blade flattening. Curr Biol in press

\section{SUPPORTING INFORMATION}

Additional Supporting Information may be found online in the supporting information tab for this article: http:// onlinelibrary.wiley.com/doi/10.1111/jipb.12998/suppinfo

Figure S1. Leaf shape robustness is dramatically reduced in atml1-1 pdf2-1

(A) Seeding phenotype of 15-d-old Col showing normal leaf morphologies. (B-F) Seeding phenotypes of 15-dold atml1-1 pdf2-1 showing highly variable leaf morphologies. Scale bars: $500 \mu \mathrm{m}$. (G-I) Superimposed outlines of multiple individual true leaves from $\mathrm{Col}(\mathbf{G})$ and atml1-1 pdf2-1 (I), showing the shape variability in the horizontal direction. The median outlines of leaf shapes are represented as blue and red lines for Col and atml1-1 pdf2-1, respectively. Quantification of leaf shape variability in Col and atml1-1 pdf2-1 is shown in
(H). Values are mean $\pm S D . * * * P<0.001$ by student's t-test. $n=52$ for Col, $n=68$ for atml1-1 pdf2-1.

Figure S2. Identification of newly formed cell walls in Arabidopsis leaf primordium

(A) Agarose-gel cross section of $\mathrm{P}_{4}$ after staining with FB28 dye which specifically associates with cellulose and polysaccharides in cell walls. (B) Magnification of the inset in (A). The new cell walls have weaker staining than the old cell walls because of less FB28 deposition. (C) Measurement of FB28 fluorescence intensity in the new cell walls and old cell walls along the arrow in (B). (D) Heat map of (B), which clearly shows the difference in FB28 fluorescence intensity between new and old cell walls. Scale bars: $10 \mu \mathrm{m}$ in (A); $5 \mu \mathrm{m}$ in (B) and (D).

Figure S3. Sensitivity to key parameters

The effect of a $10 \%$ increase or $10 \%$ decrease of four indicated parameters on the variability of simulation outputs is shown for each of three situations: full epidermis restriction (A); partial epidermis restriction (B); no epidermis restriction $(C)$. Data represent mean \pm SE from 10 independent replicates.

Figure S4. Comparison of plant tissue development with varying epidermal constraint (spring constant of outer cell wall) strength levels

For all the simulations shown, the initial tissue was rectangular in shape (the same as Figure $2 \mathrm{G}$ ). Decreasing tensile resistance strength leads to unstable tissue growth (compare $\mathbf{A}$ with $\mathbf{B}$ ), and increasing tensile resistance strength significantly inhibits or even terminates tissue growth (compare C with B).

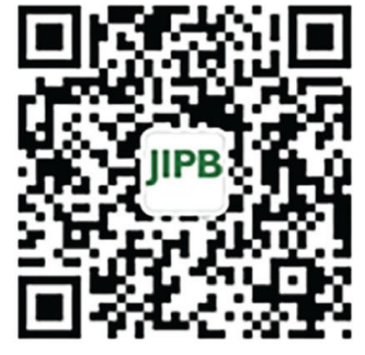

Scan using WeChat with your smartphone to view JIPB online

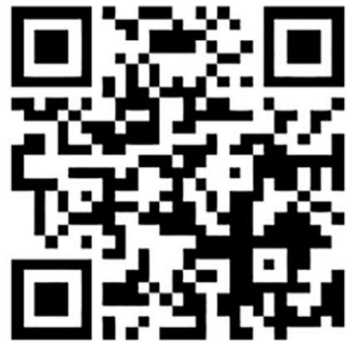

Scan with iPhone or iPad to view JIPB online 